\title{
7 Jugendforensik, Jugendhilfe und Jugendgerichtsbarkeit in den Niederlanden - Neuere Entwicklungen
}

\author{
Theodore A.H. Doreleijers
}

Im Laufe des 20. Jahrhunderts wurde in den Niederlanden die Versorgungsstruktur in der Kinder- und Jugendpsychiatrie und der Jugendhilfe unfangreich differenziert. Diese Differenzierung wurde vor allem dadurch bedingt, dass die Institutionen der Jugendhilfe verschiedenen Ministerien unterstellt wurden, nämlich den Ministerium für öffentliche Gesundheit, Soziales und Sport, dem Justizministerium und dem Erziehungsministerium. Es gibt somit kein eigenständiges Ministerium, das hauptsächlich für Jugendfragen zuständig ist.

Komplizierter wird dieser Situation dadurch, dass diese verschiedenen Bereiche zusätzlich aus unterschiedlichen Quellen ihre Finanzierung erhalten. Einrichtungen der Erziehungshilfe werden ebenso wie Justizeinrichtungen zentral aus dem Staatsbudget finanziert. Im Sozialwesen obliegt die Bereitstellung der Mittel dagegen den einzelnen Provinzverwaltungen (die vier größten Städte sind diesbezüglich Provinzen gleichgestellt). Die Psychiatrie erhält ihre Gelder aus einem Finanztopf für „außergewöhnliche medizinische Leistungen“, und private Krankenversicherungen tragen hier ergänzend die Kosten.

Zusätzlich zu dieser Differenzierung bestand über mehr als ein Jahrhundert hinweg eine subsidiäre Aufteilung der Leistungen der Jugendhilfe. Die Konkurrenz der Religionsgemeinschaften spiegelt sich heute noch in der Vielfalt von Organisationen in Politik, Erziehung, Gesundheitspflege, Medien, Sportverei- 
7 Jugendforensik, Jugendhilfe und Jugendgerichtsbarkeit in den Niederlanden Neuere Entwicklungen

nen, Theater etc. wider. So bestehen in größeren Städten römisch-katholische, jüdische, moslemische und eine Vielzahl von protestantischen Schulen. Eine ähnliche Vielfalt findet sich auf dem Gebiete der Gesundheitspflege, bei Sportvereinen, oder sogar bei Radio- und Fernsehsendern. Ähnliches galt für den Bereich der Psychiatrie und des Sozialwesens, denn auch hier existierte ein reichhaltiges Angebot an Einrichtungen neben staatlichen Institutionen.

In Städten wie Den Haag (400.ooo Einwohner) boten in den 8oer Jahren insgesamt etwa 50 Jugendhilfeeinrichtungen Leistungen an (Kriseninterventionszentren, Heime für weggelaufene Mädchen, medizinische Tageskliniken, kinder- und jugendpsychiatrische Zentren, die Bewährungshilfe für Jugendliche, Vormundschaftsbehörden etc). Jeder dieser Dienste wurde von den Trägern nicht nur finanziert, sondern es fand auch abhängig vom weltanschaulichen Hintergrund des Trägers eine inhaltliche Beeinflussung statt. Die Einrichtungen waren weder vernetzt, noch gab es einheitliche Konzepte und Angebote im Bereich der Jugendhilfe. Eine Zusammenarbeit der Jugendhilfeeinrichtungen mit den Kliniken der Kinder- und Jugendpsychiatrie bestand nicht. Aus diesem Grund waren Zuweisungen, Behandlung und Nachsorge nicht vernetzt organisiert und insgesamt herrschte wenig professionelles Arbeiten in den einzelnen Einrichtung vor, weil jeder Anbieter sein individuelles und auf die Einrichtung zentriertes Angebot ohne Kooperation mit den anderen pflegte.

1994 beschlossen die damaligen Staatssekretariate der Justiz, der Gesundheit, des Sozialen und des Sports in diesem „Institutionsdschungel“ gründlich zu reformieren. Sie initiierten ein „Megaprojekt“ mit dem Namen Regie der Jugendhilfe (Regie in de Jeugdzorg 1994). Das Hauptziel der Jugendhilfe sollte eine zweispurige Neuausrichtung sein. Der Zugang zu den Hilfeleistungen sollte durch standardisierte Verfahren bei der Aufnahme und auf den Angebots- und Versorgungsbedarf bezogene Selektion (Triage) verbessert werden. Erst wird eine Indikation für eine spezifische Jugendhilfemaßnahme gestellt, in einem zweiten Schritt wird ein Behandlungsplan entwickelt und danach wird die Jugendhilfemaßnahme umgesetzt.

\subsection{Zuweisung zur Jugendhilfe}

Um Kinder und ihre Eltern davon zu bewahren, von Ort zu Ort gesandt zu werden, wurde eine so genannte Institution mit dem Namen „einzige Tür“ eingerichtet. Hier findet die Triage statt, um mittels angemessener Kriterien die geeignete Einrichtung für das Kind und den Jugendlichen auszusuchen. In kleineren Orten und Regionen ist damit wörtlich die „Tür“ der einzigen Triagestelle der Jugendhilfe gemeint, in größeren Städten und Regionen verteilen sich diese Zugangswege auf die verschiedenen Einrichtungen. In diesen Jugendhilfeeinrichtungen wurden Aufnahmeteams aus verschiedenen Berufsgruppen aufgestellt, die in der Lage sind, nach einem Screening eine an- 
gemessene Begutachtung durchzuführen(Yperen u. Geffen 1997). Mit der aus der Begutachtung gewonnenen Einschätzung der Problemlagen der Jugendlichen soll eine Differierung für die Zuteilung zu den Maßnahmen und eine Bestimmung des erforderlichen Umfangs gewährleistet werden. Ein spezielles auf einem qualifizierten und multidisziplinären Team beruhendes Einschätzungsverfahren kann die Indikationen über Länge, Umfang, Art der Einrichtung und die Notwendigkeit weitergehender Hilfsangebote (z.B. Psychotherapie oder [teil-]stationäre Behandlung) stellen. In den letzten Jahren wurden in verschiedenen Regionen mit dieser Organisationsstruktur der Jugendhilfe Probeläufe durchgeführt, bei denen einige Anfangsschwierigkeiten zutage traten.

Sozialarbeiter der Vormundschaftsbehörden, Psychologen der Jugendpsychiatrieeinrichtungen, Bewährungshelfer für Jugendliche und Jugendarbeiter bilden gemeinsam diese Erstaufnahmeteams für das Screening und die diagnostische Begutachtung. Vor ca. zwanzig Jahren wurden ambulante psychiatrische Einrichtungen auf regionaler Basis zu sogenannten „Riaggs“ (regionale Polikliniken) zusammengefasst, nachdem sie historisch im Laufe des zwanzigsten Jahrhunderts auf konfessioneller Basis aufgebaut worden waren. Die traditionelle Einstellung, dass der „zwischen zwei Köpfen verschiedener Konfessionen auf einem Kopfkissen immer auch der Teufel liege" konnte in den achtziger Jahren allmählich überwunden werden. Bei der Arbeit der neu strukturierten psychosozialen Einrichtungen sind allerdings neue „Teufel“ geschaffen worden, die aufs Neue entschlossen bekämpft werden müssen.

Auch der Gesetzgeber konnte bisher nicht alle neuen Probleme lösen. Die Ratifikation des neuen Jugendhilfegesetzes wurde über Jahre hinweg verzögert. Ein Ziel dieses Gesetzes war es, dass alle Dienste aus der gleichen Hand finanziert werden sollten; vor allem von Kinder- und Jugendpsychiatern ging jedoch eine starke Opposition aus und es ist ihnen anscheinend gelungen, eine separate Finanzierung zu behalten.

\subsection{Programme der Jugendhilfe}

Wie oben erwähnt, hat das Projekt „regie in de jeugdzorg“ zwei Hauptziele: den Zugang zur Jugendhilfe und die Planung der Hilfestellungen zu entwickeln und zu koordinieren. Ein weiteres Ziel besteht darin, die Planung der Hilfsprogramme zu zentralisieren, die Zuweisung der Hilfen zu vereinfachen und mit dem gemeinsamen Wissen der Fachleute aus den jeweiligen Fachdisziplinen den Bedürfnissen der Kinder und ihrer Eltern besser gerecht zu werden. Bis jetzt konnte es vorkommen, dass ein psychisch schwer auffälliges Kind im Sozialwesen ungenügend diagnostiziert und behandelt wurde, danach zum öffentlichen Gesundheitsdienst (dort arbeiten nicht in jedem Fall Kinder- und Jugendpsychiater) überwiesen wurde und dadurch erst im dritten Schritt zum Kinder- und Jugendpsychiater kam. Jugendliche Straffällige fan- 
7 Jugendforensik, Jugendhilfe und Jugendgerichtsbarkeit in den Niederlanden Neuere Entwicklungen

den erst über den Umweg über die Polizei, die Kinderschutzbehörde, den Bewährungshelfer für Jugendliche und ggf. sogar noch das Gefängnis zum forensisch qualifizierten Jugendpsychiater. So konnte im Sozialwesen jahrelang ein Jugendlicher betreut werden, ohne dass ein Psychologe oder Psychiater hinzu gezogen worden ist. Auf diese Weise wurden viele Störungen nicht diagnostisch erfasst und in vielen Fällen setzte eine angemessene Behandlungen viel zu spät ein. Die vorgesehene Koordinierung der Hilfsangebote bietet für dieses Problem eine Lösung. In vielen Städten werden spezialisierte Fachgruppen für bestimmte Störungsbilder aufgebaut. In Den Haag arbeiten z.B. Mitarbeiter des Sozialwesens aus verschiedenen Bereichen (Justiz, Jugendsozialwesen, psychiatrisch-psychologische Dienste) zusammen, um Behandlungsangebote für jugendliche Straffällige mit Aufmerksamkeits-Hyperaktivitätsstörungen und autistische Kinder zu entwickeln.

Die Koordination der Behandlungsangebote muss von allen beteiligten Institutionen gemeinsam getragen werden und wird durch einen erfahrenen ,jackof-all-trades“ (Koordinator) repräsentiert. Dieser Koordinator ist der Vorsitzende eines Behandlungs-Koordinierungskomitees, der sich aus Mitgliedern verschiedener Disziplinen der beteiligten Institutionen zusammensetzt (Doreleijers 1998). Diese bilden mit ihren Leitern den Vorstand und unterstützen den Koordinator. Wenn die erweiterte Begutachtung abgeschlossen ist, wird der Fall in diesem Komitee vorgestellt. Hier wird ein „maßgeschneiderter“ Behandlungsplan entworfen, dessen einzelne Elemente (Module) werden von den verschiedenen beteiligten Institutionen durchgeführt werden. Die Fallführung liegt wiederum in den Händen eines „Case managers“ (Fallverantwortlichen), der zentral den Kontakt zu den Patienten und ihren Familie, sowie zu den beteiligten Fachinstanzen gewährleistet und somit einen zentralen Knoten im Netzwerk bildet.

\section{Fallbeispiel}

Der 15-jährige Stefan leidet an einer Aufmerksamkeits-Hyperaktivitätsstörung (ADHS) und raucht exzessiv Cannabis, vermutlich im Sinne einer Selbstmedikation. Er stammt aus einer dysfunktionalen Familie. Sein Vater leidet an ausgeprägten Symptomen des ADHS und die Mutter hat kontinuierlich Konflikte mit ihrem impulsiven und dominanten Mann. Stefan beging zunehmend schwerere Einbrüche, um seinen Suchtmittelkonsum zu finanzieren. Die Behörde ordnete eine forensisch psychologisch-psychiatrische Begutachtung an, nach deren Empfehlung eine Bewährungsstrafe vom Gericht angeordnet. Die Auflage sah vor, dass Stefan sich einer Behandlung zu unterziehen habe. Zusätzlich musste der Bewährungshelfer des Jugendlichen regelmäßig dem Staatsanwalt Bericht über sein Verhalten erstatten. Das Behandlungsprogramm bestand aus den folgenden Maßnahmen und Therapien:

1. Medikamentöse Behandlung durch einen Kinderpsychiater im lokalen Zentrum für Kinder und Jugendpsychiatrie 
2. Gruppentherapie mit dem Schwerpunkt der Suchtbehandlung wegen der Cannabisabhängigkeit und zur Verbesserung der sozialen Fertigkeiten

3. Elternberatung abwechselnd mit Familieninterventionssitzungen mit allen Beteiligenten aus der Familie

Stefan absolviert seine Gruppentherapie in der lokalen Poliklinik (Riagg). Die Elternberatung wird durch die Vormundschaftsbehörde durchgeführt, die auch für die Bewährungshilfe verantwortlich ist. Der Fallverantwortliche steht in regelmäßig in festem Intervall mit der Schule und den anderen an dem Hilfeprogramm beteiligten Fachleuten in Verbindung.

Der Erfolg eines solchen Vorgehens hängt stark vom Engagement der Leitung der beteiligten Institutionen ab, aber auch von den finanziellen Zuwendungen (Bonusleistungen), die für eine gute Kooperation aller beteiligten Hilfsinstitutionen angeboten werden. Die regelmäßige Auswertung der Erfahrungen aller beteiligten Klienten gibt der Programmkommission und den Fachpersonen in den Einrichtungen genaue Rückmeldung über den Entwicklungsstand der Jugendlichen in der Maßnahme. Auf der anderen Seite ist durch die Budgetverantwortlichen der Regierung dafür zu sorgen, dass keine Unterbrechungen im Programm auftreten.

\subsection{Haftanstalten für Jugendliche}

Hier sollen die letzten Entwicklungen in den Haftanstalten für Jugendliche in Holland beschrieben werden. Zuvor wird zum besseren Verständnis das Vorgehen nach dem holländischen Jugendstrafgesetz vorgestellt.

In Holland gilt für Jugendliche vom 12. bis zum 18. Lebensjahr das Jugendstrafgesetz. Kinder unter 12 sind vor Strafverfolgung freigestellt. Die Kinderschutzbehörde fordert vom Jugendgericht im Fall krimineller Handlungen die Einleitung einer zivilen auf Hilfe und Behandlung ausgerichteten Maßnahme für die Kinder. Das Jugendstrafrecht hat einen präventiven und pädagogischen Charakter: Nicht der Konflikt zwischen dem Handeln des Tatverdächtigen und rechtlichen Erwartung der Gesellschaft steht im Vordergrund, sondern die Suche nach geeigneten Hilfen und Problemlösungen für die psychosozialen Auffälligkeiten der Jugendlichen steht im Vordergrund.

1995 trat das revidierte Jugendstrafgesetz in Kraft. Es wurde geschaffen, um dem den gestiegenen Erwartungen der Jugendlichen nach mehr Freiheiten zu entsprechen und um gleichzeitig der politischen Forderung nach einer konsistenteren und wirkungsvolleren Antwort auf die Jugendkriminalität nachzukommen. Unter dem neuen Gesetz wurden die Höchstgrenzen für die Haftstrafen von 6 auf 12 Monate für 12-15-Jährige bzw. bis zu 24 Monaten für 16 und 17-Jährige angehoben. Die maximale Geldstrafe wurde von ca. $200 €$ auf $2.000 €$ (Stand im Jahre 20oo) erhöht. Zudem wurde ein komplexes System von Alter- 
7 Jugendforensik, Jugendhilfe und Jugendgerichtsbarkeit in den Niederlanden Neuere Entwicklungen

nativmaßnahmen zu Haft- und Geldstrafen entwickelt. Jetzt dürfen die Jugendgerichte keine Maßnahmen anstelle einer strafrechtlichen Sanktion für Jugendliche über 12 Jahren aussprechen.

Es werden ca. 70\% der von der Polizei verhafteten Jugendlichen wurden wegen der Deliktsschwere dem Staatsanwalt vorgeführt. Aber nur in 15\% aller Verhaftungen findet auf Antrag des Staatsanwaltes ein Gerichtsprozess statt. Das Jugendgericht ist nur für Fälle mit einer bereits fortgeschrittenen kriminellen Karriere vorgesehen. Aus drei verschiedenen Gründen können Jugendliche in einer Haftanstalt untergebracht werden. Die Haft hat dementsprechend drei verschiedene Funktionen:

1. Untersuchungshaft für Jugendliche, bei denen noch kein Urteil gefällt wurde. Mit diesen Jugendlichen werden ggf. diagnostische fachliche Begutachtungen in der Haftanstalt durchgeführt, wodurch sich z.T. ein anderer Haftgrund ergibt.

2. Verbüßung von Haftstrafen bei Jugendlichen.

3. In einigen Hafteinrichtungen werden auch Maßnahmen und Behandlungen durchgeführt. Diese werden als dann Maßnahmen anstelle oder begleitend zum Haftaufenthalt durchgeführt, wenn die Empfehlung aus der Begutachtung eine schwere psychische Störung oder Geistesschwäche nachwies, die einen Aufenthalt in einer geschlossenen Einrichtung notwendig macht. Auch die Maßnahmen und Behandlungen in den Haftanstalten können die Rehabilitation zu Ziel haben.

In den Niederlanden findet eine intensive Debatte darüber statt, wie diese Maßnahmen und die Behandlung in Haftanstalten gestaltet sein sollen. In dieser Debatte stehen die folgenden Punkte im Vordergrund:

1. Der Staatsanwalt und/oder eine andere Behörde verlangen eine Begutachtung nach der Inhaftierung. Sie leiten diese eigenständig ein, aber sie können aber auch die Kinderschutzbehörde hinzuziehen. Die Kinderschutzbehörde muss in Holland alle von der Polizei verhafteten eines Verbrechens verdächtigten Minderjährigen betreuen. Das Gericht kann auch eine Begutachtung durch niedergelassene oder andere ambulant tätige Fachpersonen aus Psychologie oder Psychiatrie durchführen lassen (Doreleijers 1995). Wenn die Jugendlichen in Untersuchungshaft sind, kann die Justizbehörde verlangen, dass sie in der Haftanstalt untersucht werden. Auch die Gefängnisleitung kann angefragt werden, einen Bericht von den dort tätigen Fachleuten aus den Fachbereichen Psychologie, Psychiatrie und Sozialarbeit erstellen zu lassen. Im ersten Fall wird das Gutachten recht rasch und verhältnismäßig günstig erstellt, beinhaltet aber meist nur die Sicht einer einzigen Disziplin (Schnappschuss). Im zweiten Fall ist die Beobachtungszeit länger (meist mehrere Wochen), der Bericht wird aus multidisziplinärer Perspektive erstellt und beinhaltet deshalb Verlaufsbeobachtungen des Verhaltens und der Entwicklung eines Jugendlichen während der Beobachtungszeit. Diese zweite Form der Gut- 
achtenerstellung ist jedoch 5-10 mal so teurer wie das vom externen Gutachter erstellte Gutachten.Die wesentliche Frage besteht jedoch nicht darin, welche Art des Gutachtens besser sei, sondern eher darin, wie sich die Indikationsstellung für die Erstellung eines Gutachtens vereinfachen, also standardisieren lässt. In einigen Fällen entscheidet die Art des Verbrechens für die Form und den Umfang der Begutachtung (ernsthafte Gewalt- und Sexualverbrechen werden meist eine interne umfangreiche Begutachtung erfordern, während minderschweres Fehlverhalten durch einen externen Gutachter beurteilt werden kann). Die Frage bleibt allerdings offen, wer darüber entscheiden kann, welche Form der Begutachtung erforderlich ist, wenn aus der Art des begangenen Deliktes keinerlei sichere Hinweise über eine mögliche Psychopathologie vorliegen.

2. Ein weiteres Problem ist die Frage, ob in dem Gutachten über die Beantwortung der von der Justizbehörde gestellten Fragen hinaus auch ein Maßnahme- und Behandlungsplan für die Phase nach der Verhandlung entwickelt und empfohlen werden darf. Die Anforderungen an die Diagnostik sind unterschiedlich, abhängig davon, ob das Gutachten von den Justizbehörden oder den Sozialbehörden verwandt werden soll. Die verschiedenen Parteien erwarten Unterschiedliches vom Gutachten. In diesem Spannungsfeld stehen die Bedürfnisse der Justizbehörde auf der einen Seite und den Bedürfnissen der Eltern und professionellen Helfer auf der anderen Seite gegenüber.

3. Auf der aktuellen Organisationsstufe ist noch nicht geklärt, welche Instanz während der Inhaftierung für die regelmäßige Betreuung der Jugendlichen zuständig ist. Wenn ein Psychologe auf einer Beobachtungsabteilung für das Gutachten zur im Auftrag der Justiz verantwortlich ist, kann er nicht gleichzeitig für die psychologische und therapeutische Betreuung des Jugendlichen verantwortlich sein. Die Befürworter einer Begutachtung durch Gutachter, die nicht in der Einrichtung tätig sind, plädieren dafür, ohne Ausnahme vollständig die Vergabe aller Gutachtenaufträge an einrichtungsfremde Gutachter vorzunehmen. Diejenigen, die einer einrichtungsinternen Begutachtung den Vorzug geben argumentieren, dass bei einer einzigen Konsultation pro Woche die ganze Fülle der in Dokumentation des Verlaufs gesammelten Beobachtungen verloren gehe. Um dies zu berücksichtigen, wurde in einem Gefängnis ein so genanntes „fliegendes Team“ geschaffen. Dieses arbeitet unabhängig vom Betreuungsteam und hat keine therapeutischen Funktionen bei den zu begutachtenden Jugendlichen. Sie können problemlos an regelmäßigen Fallbesprechungen teilnehmen, wenn die Jugendlichen und ihre Eltern die Erlaubnis dafür erteilen.

4. Ein weiterer Konfliktbereich ist das Verhältnis zwischen den Therapeuten der Jugendlichen in den Institutionen und den Ärzten und Pflegepersonen des medizinischen Teams. Die Therapeuten gehörendem sozialtherapeutischen und psychotherapeutischen Behandlungsteam an. Ihre Aufgabe besteht darin, das Verhalten der Jugendlichen positiv zu 
7 Jugendforensik, Jugendhilfe und Jugendgerichtsbarkeit in den Niederlanden Neuere Entwicklungen

beeinflussen, um psychische Symptome und Störungen des Sozialverhaltens zu verbessern. Das medizinische Personal ist für die somatische und psychiatrische Versorgung verantwortlich.Interessenkonflikte entstehen z.B. dann, wenn ein Jugendlicher zur Somatisierungen neigt, deswegen somatisch durch das Behandlungsteam behandelt und ggf. von der den Cruppen- und Einzelmaßnahmen befreit wird, und dadurch seine Verpflichtung zur Teilnahme an den sozialtherapeutischen und psychotherapeutischen Angeboten umgeht.

Aus dieser Beschreibung der Situation wird deutlich, dass diese Debatte über die Entwicklung der Jugendhilfe bei forensischen Maßnahmen in den Niederlanden noch lange nicht abgeschlossen ist. Das Justizministerium hat zur Klärung dieser Fragen zwei Arbeitsgruppen eingesetzt.

\subsection{Neue Einrichtungen}

In den vergangenen drei Jahren wurde in Holland eine Reihe von innovativen Verbesserungen im Bereich der Jugendhilfe, in der Justiz, vor allem im Bereich der stationären Behandlung von jugendlichen mit sozialen Auffälligkeiten und Delinquenz realisiert. Die Veränderungen resultierten aus der Erkenntnis, dass die traditionelle Inhaftierung die Prognose nicht verbessern kann, also die Rückfälle nicht verhindern kann und eine stagnierende psychosoziale Entwicklung nicht wieder in Gang bringen kann, unabhängig davon, wie professionell die Angebote in Erziehung und Ausbildung durchgeführt werden. Während die Einrichtungen weiterhin das „Bedürfnis der Gesellschaft“ nach „Vergeltung“ gegenüber den jugendlichen Straftätern befriedigen, zeigt die Forschung, dass die klassische Form der Bestrafung keine sozial ausreichende und wirtschaftlich angemessene präventive Wirkung hinsichtlich zukünftiger Straftaten zeigt und die Rückfallrate durch die klassische Form des Strafvollzuges relativ unbeeinflusst bleibt.

Deshalb wird zunehmend mehr in die Früherkennung von Warnsignalen für kriminelles Verhalten investiert. Mit delinquentem Verhalten assoziierte psychische Störungen sollen behandelt werden, die Familienprobleme gemindert und die Lern- und Verhaltensstörungen in der Schule gebessert werden. Dazu gehört, dass ein Beitrag zur sozialen Integration geleistet wird, indem die Institutionen der Jugendhilfe bei der Stellensuche behilflich sind, Lehrangebote vermitteln oder schaffen und die Jugendlichen darin unterstützen, soziale Kompetenzen und eine angemessene Eigenständigkeit zu entwickeln. Wenn diese Ziele eine Aussetzung des Vollzuges der Haft auf einige Monate, oder sogar nur einige Stunden am Tag erfordern, so stehen dafür neue Haftformen wie die „Halbgefangenschaft“ (nur nachts) zur Verfügung, um den Jugendlichen die Teilnahme an einem Tagesklinikangebot oder einer ambulanten Behandlung zu ermöglichen (Bartels et al. 1999). Der Nachteil dieser Form einer mit einer ambulanten Maßnahme kombinierten Haft ist, dass hierbei 
in einer so genannten zweigleisigen Finanzierung die Kosten von unterschiedliche Trägern übernommen werden: Das Justizsystem zahlt für die Aufwendungen der Haft, während das Gesundheitswesen und Krankenkassen die Behandlung finanzieren müssen. Eine einheitliche Lösung der Kostenträgerschaft für die kombinierten Haft- und Behandlungsangebote konnte bisher nicht durchgesetzt werden.

Eine große Zahl weiterer Maßnahmen der primären, sekundären und tertiären Prävention von Delinquenz bei Jugendlichen sind lokal von unterschiedlichen Trägern realisiert worden. Als Beispiel für diese Programme der Prävention sei hier das SPRINT Projekt in Amsterdam beschrieben. Es ist in den Niederlanden eines der wissenschaftlich am besten evaluierten Projekte (Slot et al. 1999).

Als Teil dieses Projektes werden die Lehrer von Kindern in der vierten Schulklasse dazu angeleitet, die zehnjährigen Kinder mit hohem Risiko für eine dissoziale und delinquente Entwicklung zu identifizieren. Diese Kinder werden mit Screeninginstrumenten untersucht und bei Bestätigung des Verdachts für ein Risiko wird mit den Eltern Kontakt aufgenommen. In einer Sitzung wird ihnen der Sachverhalt erklärt und sie werden eingeladen, an einer kontrollierten therapeutischen Studie zur Prävention krimineller Entwicklung im Jugendalter teilzunehmen.

Allerdings wird die Mehrzahl der anderen innovativen Projekte in Holland nicht wissenschaftlich evaluiert. Dadurch fällt es einerseits schwer ihre Wirksamkeit zu belegen und aus diesem Grunde ist es natürlich für die politischen Instanzen schwierig zu entscheiden, ob es sinnvoll ist, ein Projekt nach der Probephase weiter zu führen.

\subsection{Hilfsverbünde und Abläufe}

Das Ziel der Leitung der Jugendhilfe ist es, die Arbeit der verschiedenen professionellen Stellen aufeinander abzustimmen und zu verhindern, das die betroffenen Jugendlichen durch die „Stäbe des Citterrostes“ der Hilfsmaßnahmen fallen oder von einer in die nächste Maßnahme geschickt werden, ohne dass eine angemessene Behandlung stattfindet. Eines der Hauptziele der Arbeit in der Jugendforensik in der nächsten Zeit wird die Einrichtung von integrierten und verbundenen Hilfseinrichtungen sein. Diese Verbünde werden regional organisiert und alle Anbieter von Hilfsangeboten für Jugendliche sind daran beteiligt. Jeder Verbund wird ambulante und stationäre Behandlung anbieten können. Bei der Behandlungsplanung liegt der Focus auf der Kontinuität der Maßnahmen. Dies wird dadurch gefördert, dass auch die Finanzierung nur für kontinuierliche Maßnahmen und Behandlungsangebote zur Verfügung gestellt wird. Es werden nicht mehr die einzelnen Institutionen oder Abteilungen finanziert, sondern ein Budget für Behandlungsprogramme aufgestellt. Die Produktivität (und wenn möglich die Wirksamkeit) ist von 
7 Jugendforensik, Jugendhilfe und Jugendgerichtsbarkeit in den Niederlanden Neuere Entwicklungen

den Anbietern jährlich zu belegen, damit sie weiter Gelder erhalten. Evaluationsstudien in den holländischen Haftanstalten für Jugendliche haben gezeigt, dass in den letzten Jahren vor allem ein ausgeprägter Mangel in der Nachsorge für die hohen Rückfallraten verantwortlich war. Somit muss durch die Überwachung der Abläufe vor allem darauf geachtet werden, dass Jugendliche während oder bereits vor und nach ihrer Entlassung aus der Haft oder einer Maßnahme intensiv beraten und begleitet werden.

\subsection{Probleme im System der forensischen Jugendhilfe}

Diese Entwicklungen erscheinen den Beteiligten auf den ersten Blick beeindruckend zu sein. Es sind sicher besonders wichtige Innovationen und die Beteiligten gehen davon aus, dass die Jugendforensik in den Niederlanden ein „Trendsetter“ im Umgang mit der Zunahme von Gewalt, Kriminalität und Entfremdung bei den Jugendlichen sein wird. Es bleibt aber noch viel zu tun, um weitere Verbesserungen durchzusetzen. Folgende Problemstellen und Fußfallen im System sind identifiziert: Angenommen ein Jugendlicher wird in einer forensischen Tagesklinik aufgenommen und die Therapie ist dank dem Schutz der vom Gericht verordneten unfreiwilligen Behandlung erfolgreich. Zufälligerweise wird er verhaftet, weil er eine kleine Straftat begeht, beispielsweise eine CD klaut. Dies kann zur Folge haben, dass er trotz allem seine Strafe absitzen muss. Dies kann kontraproduktiv für die Durchführung der Maßnahme sein, weil die kontinuierliche Betreuung unterbrochen wird. Der Bewährungshelfer bzw. Sozialarbeiter muss sich dann für Reintegration des Jugendlichen in die Maßnahme bei Gericht einsetzen oder der Anwalt des Jugendlichen kann versuchen, dem Gericht diese besondere Lage erklären. Es fehlt jedoch noch der gesetzliche und juristische Rahmen für alle Eventualitäten im Verlauf der Maßnahmen, während die modernen Einrichtungen bereits arbeiten. Auf der Seite der Gerichte findet sich häufig die Meinung, dass die Mitarbeiter der jugendforensischen Hilfseinrichtungen ihre Arbeit ohne fundierte Kenntnisse der juristischen und gesetzlichen Grundbedingungen durchführen.

Eine weitere Schwierigkeit besteht oft darin, die Familien bzw. Eltern der Jugendlichen in die Maßnahme und Behandlung einzubeziehen. Die Eltern sind unter Umständen selber sozial oder psychisch beeinträchtigt oder delinquent auffällig und möchten in der Maßnahme nicht mitarbeiten. Viele Eltern nichtniederländischer delinquenter Jugendlicher leben im Ausland oder sind selber im Gefängnis. Manchmal geht es bei der Behandlung sogar primär um die Streitigkeiten zwischen den Eltern und die familiären und erzieherischen Funktionsstörungen. Das bestehende System stellt für diese Probleme leider noch keine geeigneten Werkzeuge und Angebote zur Verfügung. Regelmäßig stellt auch die Tatsache eine Behinderung dar, dass viele die Eltern ihr Sorgerecht behalten, obwohl sie bei den Maßnahmen nicht kooperieren. Jugendliche sind dann oft zu alt für zivilrechtliche Maßnahmen. Dadurch geht wert- 
volle Zeit verloren, denn wenn die Jugendlichen ihr siebzehntes Lebensjahr erreicht haben, hält das Gericht es oft für nicht mehr für sinnvoll, die Jugendlichen in einer Maßnahmen zu platzieren.

\subsection{Schlussfolgerungen}

Nach den durchgeführten Veränderungen beginnen in den Niederlanden die verschiedenen Fachdisziplinen, die Ministerien und die Kostenträger sich besser bei Maßnahmen der forensischen Jugendhilfe abzusprechen. Es wird zunehmend anerkannt, dass Maßnahmen für effiziente Bekämpfung der Kriminalität und eine wirksame Beeinflussung gefährdeter Jugendlicher hohe Anforderungen an die Teams und Einrichtungen stellen, die laufend durch begleitende Forschung evaluiert und daraufhin weiterentwickelt werden müssen.

\section{Literatur}

Bartels AAI, Parker Brady M, Doreleijers TAH (1999) Sociale vaardigheidstraining als basis voor een forensisch jeugdpsychiatrisch dagklinisch programma (Social Skills Training as a Basis for a Forensiv Adolescent Psychiatric Day Treatment Programm). In: Collot d’Escury-Koenigs A, van der Linden A, Snaterse T (Eds.) Van preventie tot straf. Naar meer sociale vaardigheden bij jongeren. Lisse: Swets \& Zeitlinger.

Bartels JAC (1997) Jeugdstrafrecht (Juvenile Criminal Law). In: Bartels JAC, Duits N, Gunning WB (Eds.) Jeugdpsychiatrie en Recht. Assen: Van Gorcum.

Boendenmaker L (1999) Justitele behandelinrichtingen voor jongeren. Populatie en werkwijze (Judicial Treatment Centers for young people. Population and treatment methods). Leuven/Apeldoorn: Garant.

Doreleijers TAH (1995) Diagnostiek tussen Jeugdstrafrecht en Hulpverlening (Diagnostic Assessment between the Juvenile Criminal Law and Professional Assistence). Arnhem: Gouda Quint.

Doreleijers TAH (1998) Het netwerk sluit zich. Over samenwerking in de aanpak van jeugdige delinquenten met stornissen (A closing network. About cooperation in the approach of disordered juvenile delinquents). In: Koops W, Slot NW (Eds.) Van Lastig tot misladig. Houten/Diegem: Bohn Stafleu Van Loghum.

Herk L van (1997) De Justitele Jeugdinrichtingen (Juvenile Detention Centers). In: Bartels JAC, Duits N, Gunning WB (Eds.) Jeugdpsychiatrie en Recht. Assen: Van Gorcum.

Regie in de Jeugdzorg (Direction in Youth Care) (1994) Standpunt van de ministers van Welzijn, Volksgezondheid en Cultuur en Justitie (Point of View of the Ministers of HWS and Justice). Den Haag: Ministerie van Welzijn, Volksgezonheid en Cultuur.

Slot NW, Duivenvoorden Y van, Orobio de Castro B, Speekenbrink M, Afkerin A (1999) Voortgang van het project Psrint. Deelrapport II (Proceeding Report of the Sprint Project. Part II.). Amsterdam/Duivenrecht: PI Research.

Yperen TA, Geffen L (1997) Screening, diagnostiek en indicatiestelling. Toegang tot de jeugdzorg (Screening, diagnostics and indication. Access to Youth Care). Den Haag: Ministerie van Volksgezondheid, Welzjin en Sport. 